\title{
How does the initial free volume distribution affect shear band formation in metallic glass?
}

\author{
CHEN Yan ${ }^{1}$, JIANG MinQiang $^{1,2} \&$ DAI LanHong ${ }^{1,2^{*}}$ \\ ${ }^{1}$ State Key Laboratory of Nonlinear Mechanics, Institute of Mechanics, Chinese Academy of Sciences, Beijing 100190, China; \\ ${ }^{2}$ State Key Laboratory of Explosion Science and Technology, Beijing Institute of Technology, Beijing 100081, China
}

Received March 15, 2011; accepted May 16, 2011; published online June 14, 2011

\begin{abstract}
Introducing heterogeneities into the structure is an effective way to enhance the plasticity in metallic glasses (MGs). As natural heterogeneity, the original randomly distributed free volume in MGs has been found to be in favor of plasticity. However, the exact correlation between the free volume distribution and mechanical response is still unclear. In this paper, we investigate the shear banding in MGs with different structural disorders, characterized by both the free volume concentration (FVC) and the free volume dispersion (FVD). It is found that, either high FVC or wide FVD leads to low activation stress of shear band; wide FVD promotes the multiplication of shear bands but high FVC restricts it. It reveals that the yield strength in MGs is dependent on the amount of free volume while the plasticity mainly relies on the distribution. An optimum combination of the two aspects probably helps to design a MG of both good plasticity and high strength.
\end{abstract}

metallic glass, free volume, shear band, yield strength

PACS: 61.43. Dq, 61. 43. Bn, 62. 20. Fe, 81. 05. Kf

\section{Introduction}

Metallic glasses (MGs) have exhibited potential as structural materials due to their outstanding range of mechanical properties [1-4] although structural applications are currently impeded by the limited plasticity at room temperature. Due to the absence of long-range order or dislocation-like defects as in crystalline alloys, MGs fail by forming intense shear bands which propagate catastrophically without work hardening [5-13]. In recent years, several effective ways to improve the plasticity of MGs have been developed, such as the introduction of the residual stress [14], the optimum design of material composites [15-18] and the presence of heterogeneities by nano- or micro-particles and/or phases separation [19-21]. These methods produce heterogeneous structures within MGs which act as initiation sites for the

*Corresponding author (email: lhdai@Inm.imech.ac.cn) formation of shear bands and also a barrier to the rapid propagation of shear bands. Therefore, instead of several dominant shear bands, the multiplication of shear bands is observed in the modified MGs of good plasticity. Indeed, MGs are inherently heterogeneous because of the randomly distributed free volume, which can be the potential sites of shear process. We may expect that by controlling the free volume, large plasticity can be achieved in MGs. Recently, Chen et al. $[18,22]$ reported the enhancement of plasticity in monolithic MGs attributed to a large amount of randomly distributed free volume induced by minor alloying or a high cooling rate. Jiang and Dai [23] also found that the atomic structure with large packing dispersion within a MG leads to a bigger bulk-shear modulus ratio in favor of its global plasticity. However, a clear relation between the initial free volume and the mechanical response is still unknown. In this paper, based on the widely accepted free volume model $[5,10,24-26]$, we investigate the shear banding behavior in MG samples with different disordered structures by numer- 
ical simulations. Not only the free volume concentration (FVC) but also the free volume dispersion (FVD) is found to exert a critical influence on the global plasticity and yield resistance. The underlying correlations revealed here would provide clues to designing ductile MGs.

\section{Theoretical framework}

The free volume dynamics has been found to be critical to the plastic flow in MGs, and the rearrangement of atoms accompanying the creation and annihilation of free volume contributes to local shearing deformation [27-30]. In 1977, Spaepen developed a steady-state inhomogeneous flow model based on a competition between stress-driven creation and diffusion annihilation of free volume for MGs [5]. In Spaepen's model, both the plastic flow equation and the free-volume evolution are driven by shear stress. This framework can be extended to the generalized multiaxial stress state using the $J_{2}$ invariant of the stress tensor [24,25, 28], and the flow equation is expressed as:

$$
\dot{\varepsilon}_{i j}^{p}=\exp \left(-\frac{1}{v_{f}}\right) \sinh \left(\frac{\sigma_{e}}{\sigma_{0}}\right) \frac{s_{i j}}{\sigma_{e}},
$$

where $v_{f}=\bar{v}_{f} / \alpha v *$ is the normalized free volume with $\bar{v}_{f}$ being the mean free volume; $\alpha$ is a geometrical factor of order $1 ; v^{*}$ is the hard-sphere volume of an atom; the deviatoric stress tensor is $s_{i j}=\sigma_{i j}-\sigma_{k k} \delta_{i j} / 3$ and the effective stress is $\sigma_{e}=\sqrt{3 s_{i j} s_{i j} / 2} ; \quad \sigma_{0}=2 k_{\mathrm{B}} T / \Omega$ is the reference stress, with the Boltzmann constant $k_{\mathrm{B}}$, temperature $T$, and atomic volume $\Omega$. In addition to the plastic component of strain rate $\dot{\varepsilon}_{i j}^{p}$, the elastic component is

$$
\dot{\varepsilon}_{i j}^{e}=\frac{1+v}{E}\left(\dot{\sigma}_{i j}-\frac{v}{1+v} \dot{\sigma}_{k k} \delta_{i j}\right)
$$

as the measure of disorder, free volume is created by the applied stress squeezing an atom into a hole smaller than itself and is annihilated by a series of atomic jumps. In the multiaxial stress state, the free-volume evolution equation is given by

$$
\dot{v}_{f}=\frac{1}{\alpha} \exp \left(-\frac{1}{v_{f}}\right)\left[\frac{\sigma_{0}}{S \beta v_{f}}\left[\cosh \left(\frac{\sigma_{e}}{\sigma_{0}}\right)-1\right]-\frac{1}{n_{d}}\right],
$$

where $\beta=v^{*} / \Omega \sim 1$, the Eshelby factor $S=E / 3(1-v)$ with the Young's modulus $E$ and Poisson ratio $v$, and $n_{d}$ is the number of diffusive jumps necessary to annihilate a free volume equal to $v^{*}$, ranging between eqs. (3)-(10).

The constitutive law for MGs is described by eqs. (1)-(3). We know that the distribution of initial free volume affects significantly the deformation of MGs [10,24,27]. The in- homogeneous deformation is caused by the non-uniform distribution of free volume, which may result from quenching processes or from thermal fluctuation. It is reasonable to assume that the inhomogeneous part of free volume follows a Gaussian distribution [24,27], and then a finite amplitude disturbance in the form of a two-dimensional Gaussian function is added to the initial homogeneous distribution of free volume, i.e.

$$
v_{f 0}(x, y)=v_{0}+\delta \exp \left[-\frac{\left(x-x_{0}\right)^{2}}{\Delta_{x}{ }^{2}}-\frac{\left(y-y_{0}\right)^{2}}{\Delta_{y}{ }^{2}}\right],
$$

where $v_{0}$ is a constant; $\delta$ is the amplitude of the disturbance, measuring the FVC; $\left(x_{0}, y_{0}\right)$ is a random location within the sample; $\Delta_{x}$ and $\Delta_{y}$ are the characteristic half width, representing the FVD. The bigger $\Delta_{x}$ or $\Delta_{y}$ is, the more dispersedly or homogeneously the free volume is distributed. Based on the above theoretical framework, a numerical analysis can be carried out on the deformation of MGs for different initial free volume distributions.

\section{Simulation}

MGs show a topological disorder, and the inhomogeneous flow is found to have resulted from a dynamic competition between creation and annihilation of structural disorder or free volume [5,24,25,27-30]. To investigate quantitatively the correlation between the original structural disorder and the mechanical response of MGs, we developed MG samples with different concentrations and dispersions of the initial free volume distribution. A rectangular sample with the height $a$ and width $b$ is considered here. As shown in Table 1, all the samples have the initial homogeneous free volume $v_{0}=0.07$; Samples a1-a5 show the same amplitude of free volume disturbance $\delta=0.005$ but different FVDs, i.e., $\Delta_{x} / a$ and $\Delta_{y} / b$; Samples b1-b5 have the same FVD, but different magnitudes of FVC.

The free volume model is found effective in modeling the processes of shear bands that contribute to the macroscopic plasticity behavior [24,25]. We implemented the above constitutive in the finite-element computer program ABAQUS by writing a VUMAT subroutine. Plane-strain compression of $\mathrm{Zr}_{41.2} \mathrm{Ti}_{13.8} \mathrm{Ni}_{10} \mathrm{Cu}_{12.5} \mathrm{Be}_{22.5} \mathrm{MG}$ (Vitreloy 1) is simulated. A rectangular sample of a height-to-width ratio of 3:1 was modelled using 4800 ABAQUS-CPE4R plainstrain elements. Just because the free volume constitutive is

Table 1 MG samples with different initial free volume distributions

\begin{tabular}{cccccc}
\hline Samples $(\delta=0.005)$ & $\mathrm{a} 1$ & $\mathrm{a} 2$ & $\mathrm{a} 3$ & $\mathrm{a} 4$ & $\mathrm{a} 5$ \\
\hline$\Delta_{x} / a=\Delta_{y} / b$ & $1 / 20$ & $3 / 40$ & $1 / 10$ & $1 / 8$ & $1 / 5$ \\
\hline \hline Samples $\left(\Delta_{x} / a=\Delta_{y} / b=1 / 10\right)$ & $\mathrm{b} 1$ & $\mathrm{~b} 2$ & $\mathrm{~b} 3$ & $\mathrm{~b} 4$ & $\mathrm{~b} 5$ \\
\hline$\delta$ & 0.001 & 0.003 & 0.005 & 0.007 & 0.009 \\
\hline
\end{tabular}


length independent and the boundary condition is a constant remote strain rate, the length scale in the following contour plots of simulation results is of arbitrary unit. The mechanical and material constants are obtained as [25,31]: the Young's modulus $E=96 \mathrm{GPa}$, Poisson ratio $v=0.36, k_{\mathrm{B}}=1.38$ $\times 10^{-23} \mathrm{~J} / \mathrm{K}, \Omega=2.34 \times 10^{-29} \mathrm{~m}^{3}, T=300 \mathrm{~K}, n_{d}=3, \alpha=0.15$ and $\beta=1$.

\section{Results and discussion}

The initial free volume distributions of Samples a1-a5 are illustrated in Figure 1. Since the five samples have the same amplitude of free volume disturbance $\delta$, the obvious difference of structural disorder is due to the change of dispersion width. With the increase of $\Delta_{x}$ or $\Delta_{y}$, the free volume is distributed more randomly. Under applied compressive load, the free volume within the sample evolves greatly and coalesces into localized bands. Figure 2 shows the free volume distributions of Samples a1-a5 at the compressive strain $\varepsilon=0.1$. We see that in the samples with original dispersed free volume, such as in Samples a3-a5, a large quantity of free-volume-induced bands are formed, they intersect to each other and assemble as a web. In those bands, the high free volume concentration would lead to a significant decrease of viscosity, facilitating shear deformation.

Figure 3 plots the stress-strain curves of Samples a1-a5 under compression. The five samples show the similar trend. The materials first deform linear-elastically until it reaches the yield point. At the yielding, they exhibit little plasticity. Instead of work hardening usually observed in crystalline alloys, a strain softening is observed right after the yielding. The yield strength-to-modulus ratio $\sigma_{y} / E(\sim 0.05)$ has a reasonable order of magnitude as seen in the experimental work. We note that the five samples show a little difference on yield strengths (see the inset in Figure 3), which depends on the FVD width. With the increase of $\Delta_{x}$ or $\Delta_{y}$, the yield strength is decreasing. It means that randomly distributed

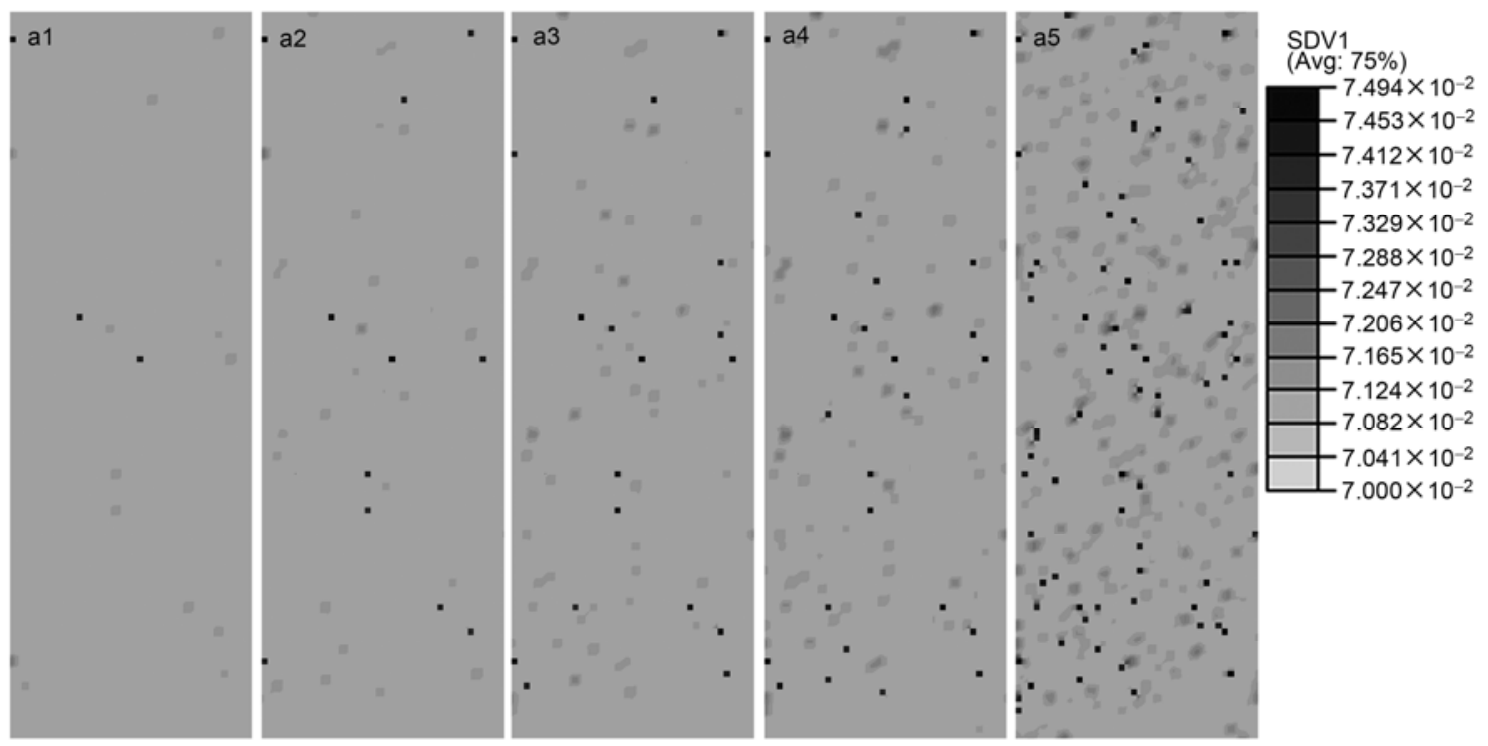

Figure 1 Initial free volume distributions of Samples a1-a5.
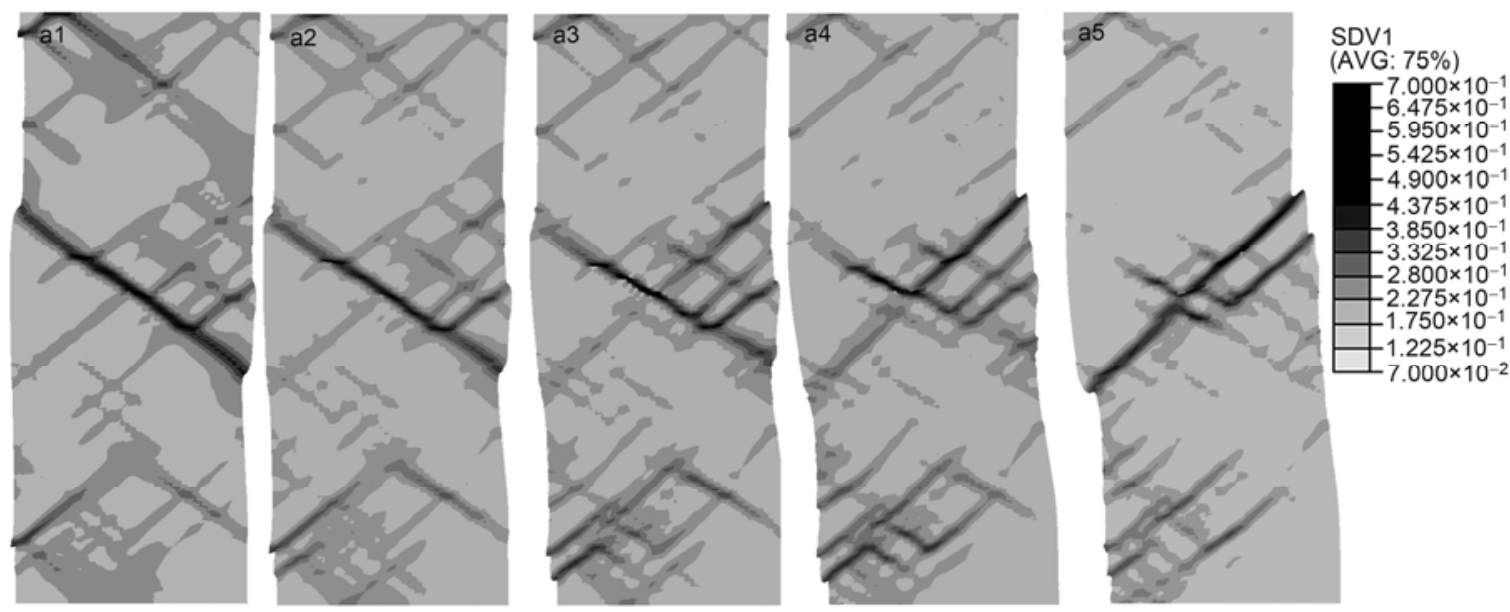

Figure 2 Free volume distributions of Samples a1-a5 at the compressive strain $\varepsilon=0.1$. 
free volume facilitates the nucleation of shear bands and then weakens the shear resistance of MGs. Moreover, we find that in the softening period, the stress of Sample a3 (see the blue curve) falls down more slowly than those of the other four samples. It may suggest that this sample can experience more plastic strain before the final failure and thus exhibit better plasticity.

From Figure 2, the initial inhomogeneous distribution of free volume can be intensified by an applied load: in the locations with high FVC, the free volume grows fast and coalesces into narrow bands. Usually, shear deformation can be easily accommodated by those weak bands due to a low viscosity, forming localized shear bands. Figure 4 displays the shear band profiles by the contour plots of the effective plastic strain fields at the compressive strain $\varepsilon=0.1$. At this strain, the stress almost reaches a steady flow. We see that with varying coefficient $\Delta_{x}$ or $\Delta_{y}$, the number and distribution of shear bands change greatly. There are few minor shear bands formed in Sample a1 besides such a major shear band that should lead to fracture. In Samples a2-a5, the dominant shear bands bifurcate and intersect with other bands, their propagation are then blocked and even completely suppressed (as in Sample a5), resulting in initiation and multiplication of new shear bands. It is indicated that shear bands are inclined to form in the structure with a great amount of free volume randomly distributed. The locations with relatively high FVC usually act as sites for shear band initiation and branching. Obviously, Samples a2-a5 have more potential sites than Sample a1, and they therefore exhibit better ductility than the latter. An interesting point is that the number of shear bands does not increase monotonously with the increase of $\Delta_{x}$ or $\Delta_{y}$. There seems to be a critical $\Delta_{x}$ or $\Delta_{y}$ which leads to an optimum structure favorable for plasticity. Take for example Sample a3 with $\Delta_{x} / a=\Delta_{y} / b=1 / 10$. It displays more intersecting shear bands than Samples a4 and a5, but shows a lower softening speed than the latter (see Figure 3). This suggests that there should
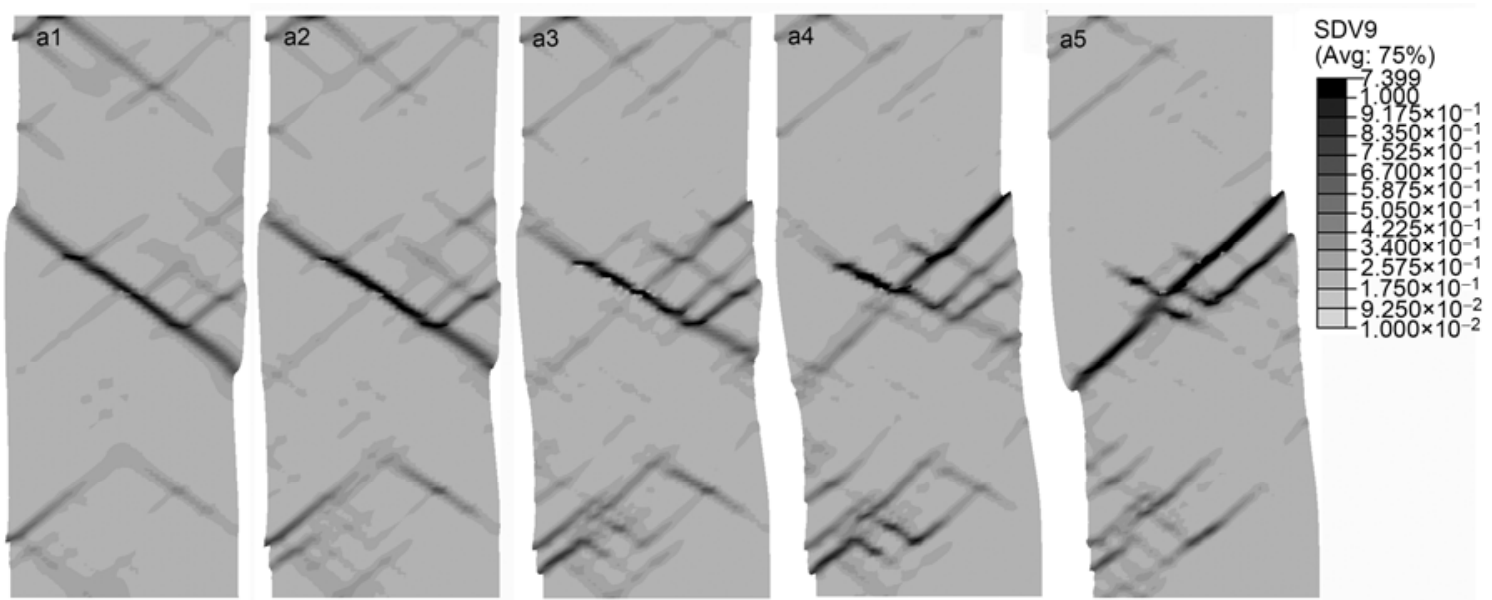

be a characteristic spacing of shear-band nucleation sites in MGs, which greatly helps the intersection among shear bands but retards their coalescence or further propagation.

Now if the initial VFD is fixed, i.e, $\Delta_{x} / a=\Delta_{y} / b=1 / 10$, what is the influence of the FVC amplitude on the mechanical response? Figure 5 shows the initial free volume distributions of Samples b1-b5. The five samples have the same free volume dispersion but different amplitudes of free volume disturbance $\delta$. At the compressive strain $\varepsilon=0.1$, the free volume displays quite different distributions in the samples as shown by Figure 6. In Sample b1, the free volume is almost homogeneous while in other samples it coalesces into localized bands. It is found that in the samples with original higher FVC, such as in Samples b4 and b5, the free-volume bands are more intense, indicating a stronger inhomogeneity of the structure.

Figure 7 gives the stress-strain curves for Samples b1-b5. It is found that the yield strength is decreased with the increase of $\delta$ (see the inset), implying that bigger FVC leads to an easier initiation of shear band. What's more, from the shear band profiles illustrated by Figure 8, we can see that,

Figure 4 Shear band profiles by the contour plots of the effective plastic strain fields at the compressive strain $\varepsilon=0.1$ for Samples a1-a5. 


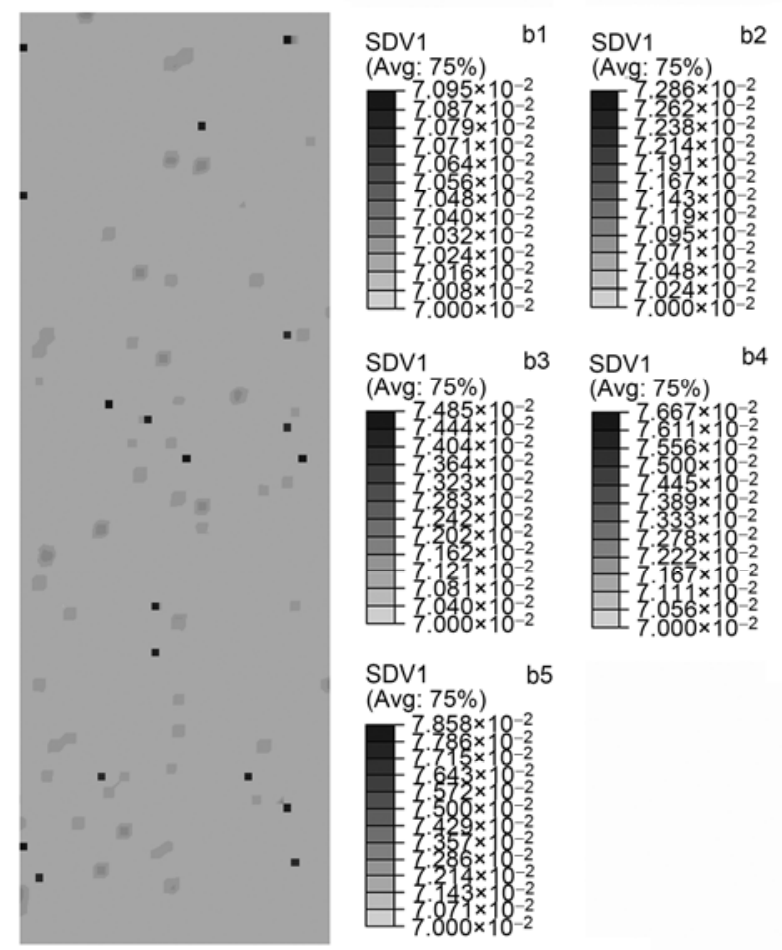

Figure 5 Initial free volume distributions of Samples b1-b5.

under the same global strain, Sample b1 shows a uniform plastic deformation, indicating a good capability of plasticity. With the increase of $\delta$, the inhomogeneous deformation becomes significant (see Samples b2-b5), which is reflected by the shear banding. Major shear bands fully developed in Samples b4 and b5 probably cause an immediate failure with little further plastic deformation. It is revealed that the sample with smaller $\delta$ can accommodate more plastic deformation in a near-homogeneous manner through profuse and concurrent shear-band formation. From the above discussions, we note that a great amount of free volume does not always correspond to a good plasticity, since increasing
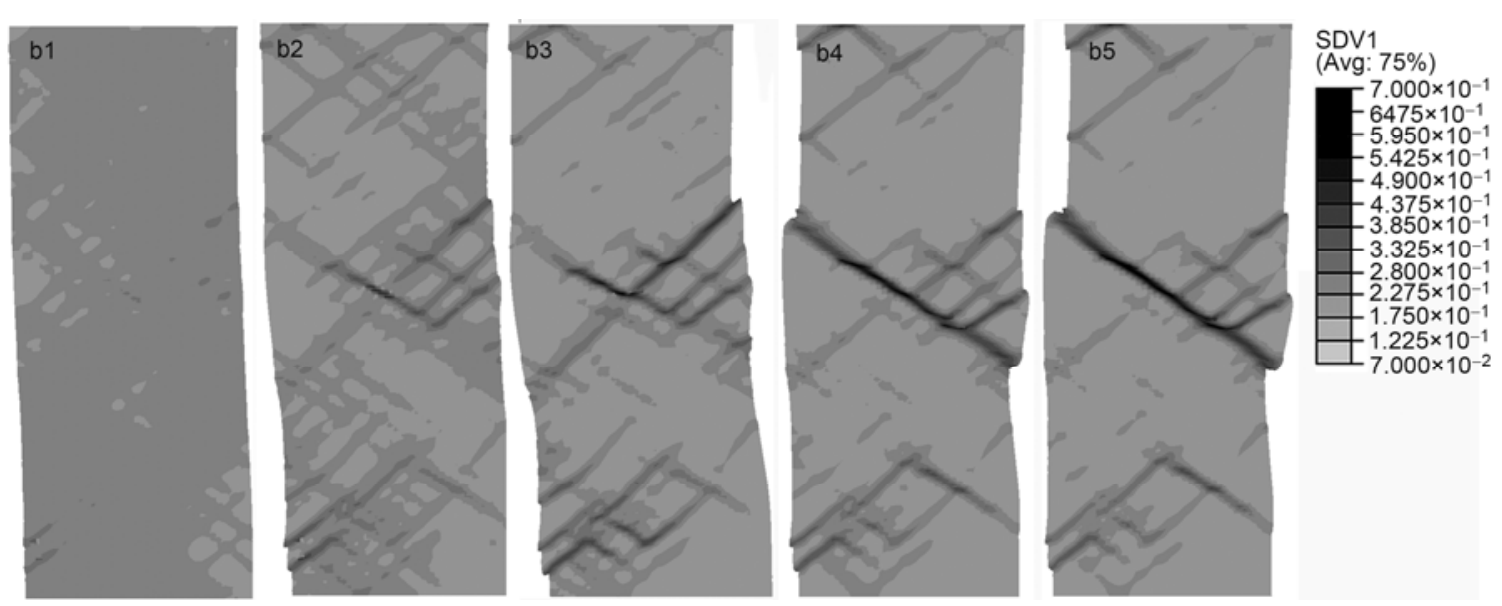

the magnitudes of FVC and FVD shows distinct effects on multiple shear banding in MGs.

It is found that the structural conditions for multiple shear banding or plasticity of MGs are low FVC and relatively wide FVD. We know that the limited plasticity in MGs can be enhanced by introducing micrometer-or nanometer-sized heterogeneities such as crystallites, and phase separation [19-21]. These heterogeneities would initiate a great number of shear bands which suppress the propagation of each other. Free volume in fact is a kind of inherent heterogeneity in MGs. The randomly distributed free volume with a critical concentration can be the sites for shear band initiation and branching. A wider FVD means more potential sites. Concurrent nucleation of shear bands together with the high branching of the band then restricts the dominant shearing process. However, as an extreme case, if the potential sites of shear banding are everywhere in a sample, small shear bands should easily nucleate and then immediately coalesce to failure. That is why a critical dispersion width is found in our simulation, in which shear bands are more inclined to intersect to each other than to coalesce. When the FVC within a MG varies largely from one position to another (i.e., higher FVC and smaller FVD), the location with higher FVC is prior to forming a dominant shear band which restrains other small shear processes. It would be adverse to the plasticity. Usually, MGs of good plasticity can be obtained by using a high cooling rate, because during the process more randomly distributed free volume or wider FVD is inherited from the liquid [15,16,22,32]. Through compositional changes, Liu et al. [17] designed super- plastic bulk metallic glasses which are composed of hard regions surrounded by soft regions. They also found that there are much more atomic-scale open volumes existing in the soft regions. This implies that the unique structure may actually result from a special non-uniform distribution of free volume. On the other hand, a large amount of free volume reduces the barrier to atomic movement, and a shear band can form easily at a low yielding point. Therefore, increas-

Figure 6 Free volume distributions of Samples b1-b5 at the compressive strain $\varepsilon=0.1$. 


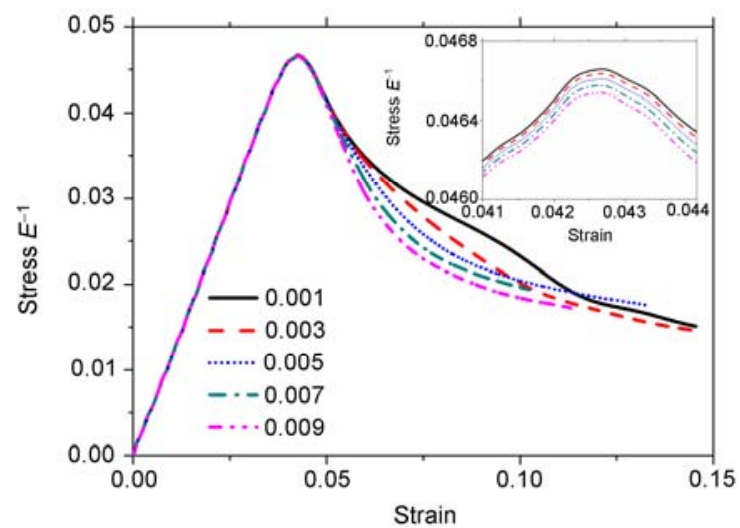

Figure 7 (Color online) Stress-strain curves of Samples b1-b5 under compression.
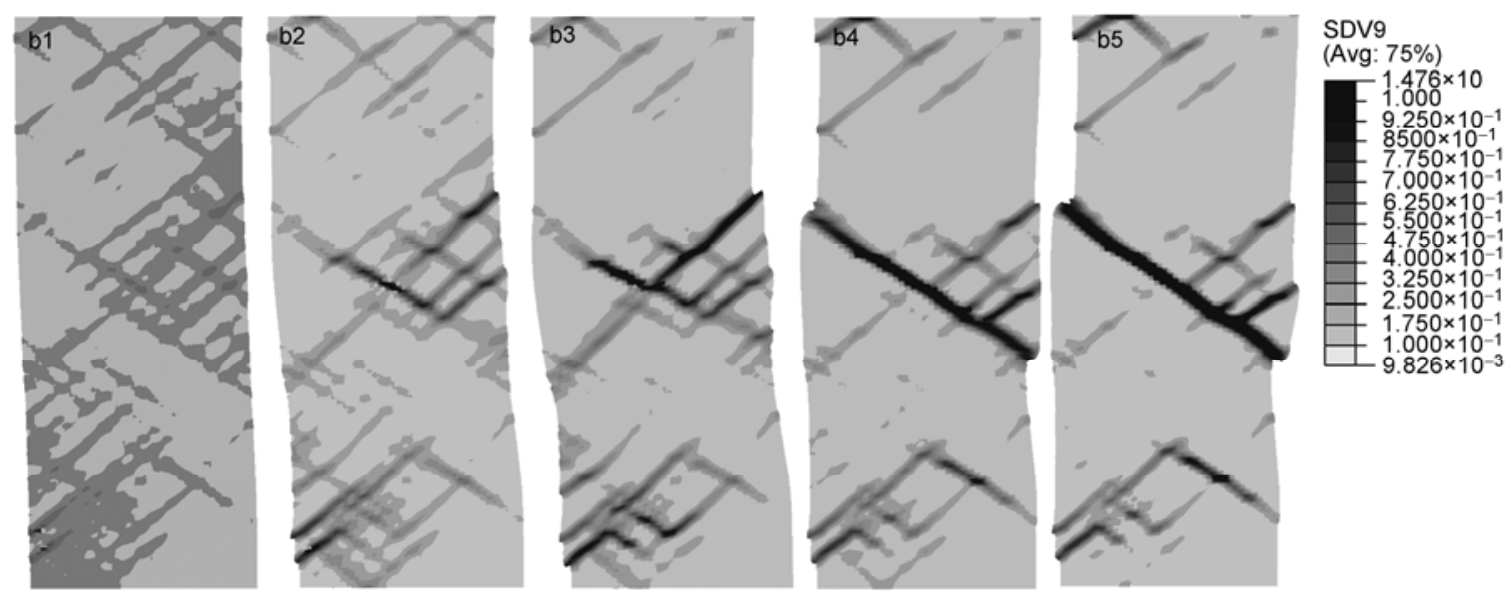

Figure 8 Shear band profiles by the contour plots of the effective plastic strain fields at the compressive strain $\varepsilon=0.1$ for Samples b1-b5.

ing either the magnitude of FVC or the dispersion leads to a decrease of activation stress of shear band in MGs.

\section{Conclusions}

In this paper, we reveal that the plasticity in MGs is not merely determined by the amount of free volume but its distribution. The FVC and FVD, which determine the initial structural disorder in MGs, have a great effect on the shear band behavior. Both high FVC and wide FVD lead to low activation stress of shear band; wide FVD promotes the multiplication of shear bands but high FVC retards it. This implies that such a MG with good plasticity and high strength can be probably obtained by controlling small FVC and relatively wide FVD. Our findings shed an insight into the micro-scale mechanisms of macroscopic mechanical behavior for MGs, providing useful clues to the design of ductile-and-stronger MGs.

This work was supported by the National Natural Science Foundation of China (Grants Nos. 10725211, 11002144, 11021262), the National Natural Science Foundation of China-NSAF (Grant No. 10976100), the National
Key Basic Research Program of China (Grant No. 2009CB724401) and the Key Project of Chinese Academy of Sciences (Grant No. KJCX2-YWM04).

1 Johnson W L. Bulk glass-forming metallic alloys: Science and technology. MRS Bull, 1999, 24: 42-56

2 Inoue A, Wang X M. Bulk amorphous FC20 (Fe-C-Si) alloys with small amounts of $\mathrm{B}$ and their crystallized structure and mechanical properties. Acta Mater, 2000, 48: 1383-1395

3 Wang W H, Dong C, Shek C H. Bulk metallic glasses. Mater Sci Eng R, 2004, 44: 45-89

4 Greer A L, Ma E. Bulk metallic glasses: at the cutting edge of metals research. MRS Bull, 2007, 32: 611-615

5 Spaepen F. A microscopic mechanism for steady state inhomogeneous flow in metallic glases. Acta Metall, 1977, 25: 407-415

6 Spaepen F. Homogeneous flow of metallic glasses: A free volume perspective. Scripta Mater, 2006, 54: 363-367

7 Schuh C A, Hufnagel T C, Ramamurty U. Mechanical behavior of amorphous alloys. Acta Mater, 2007, 55: 4067-4109

8 Chen M W. Mechanical behavior of metallic glasses: Microscopic understanding of strength and ductility. Annu Rev Mater Res, 2008, 38: 445-469

9 Dai L H, Bai Y L. Basic mechanical behaviors and mechanics of shear banding in BMGs. Int J Impact Eng, 2008, 35: 704-716

10 Jiang M Q, Dai L H. On the origin of shear banding instability in metallic glasses. J Mech Phys Solids, 2009, 57: 1267-1292 
11 Meng J X, Ling Z, Jiang M Q, et al. Dynamic fracture instability of tough bulk metallic glass. Appl Phys Lett, 2008, 92: 171909

12 Trexler M M, Thadhani N N. Mechanical properties of bulk metallic glasses. Prog Mater Sci, 2010, 55: 759-839

13 Liu L F, Dai L H, Bai Y L, et al. Comparison of shear banding in BMGs due to thermal-softening and free volume creation. Sci China Ser G-Phys Mech Astron, 2008, 51: 1367-1379

14 Zhang Y, Wang W H, Greer A L. Making metallic glasses plastic by control of residual stress. Nat Mater, 2006, 5: 857-860

15 Schroers J, Johnson W L. Ductile bulk metallic glass. Phys Rev Lett, 2004, 93: 255506

16 Das J, Tang M B, Kim K B, et al. "Work-hardenable" ductile bulk metallic glass. Phys Rev Lett, 2005, 94: 205501

17 Liu Y H, Wang G, Wang R J, et al. Super plastic bulk metallic glasses at room temperature. Science, 2007, 315: 1385-1388

18 Chen L Y, Fu Z D, Zhang G Q, et al. New class of plastic bulk metallic glass. Phys Rev Lett, 2008, 100: 075501

19 Hays C C, Kim C P, Johnson W L. Microstructure controlled shear band pattern formation and enhanced plasticity of bulk metallic glasses containing in situ formed ductile phase dendrite dispersions. Phys Rev Lett, 2000, 84: 2901-2904

20 Hofmann D C, Suh J Y, Wiest A, et al. Designing metallic glass matrix composites with high toughness and tensile ductility. Nature, 2008, 451: 1085-1089

21 Yao K F, Ruan F, Yang Y Q, et al. Superductile bulk metallic glass. Appl Phys Lett, 2006, 88: 122106

22 Chen L Y, Setyawan A D, Kato H, et al. Free-volume-induced enhancement of plasticity in a monolithic bulk metallic glass at room temperature. Scripta Mater, 2008, 59: 75-78

23 Jiang M Q, Dai L H. Short-range-order effects on intrinsic plasticity of metallic glasses. Philos Mag Lett, 2010, 90: 269-277

24 Huang R, Suo Z, Prevost J H, et al. Inhomogeneous deformation in metallic glasses. J Mech Phys Solids, 2002, 50: 1011-1027

25 Gao Y F. An implicit finite element method for simulating inhomogeneous deformation and shear bands of amorphous alloys based on the free-volume model. Modelling Simul Mater Sci Eng, 2006, 14: 1329-1345

26 Wang J G, Zhao D Q, Pan M X, et al. Correlation between onset of yielding and free volume in metallic glasses. Scripta Mater, 2010, 62: $477-480$

27 Argon A S. Plastic deformation in metallic glasses. Acta Matall, 1979, 27: 47-58

28 Steif P S, Spaepen F, Hutchinson J W. Strain localization in amorphous metals. Acta Metall, 1982, 30: 447-455

29 Falk M L, Langer J S. Dynamics of viscoplastic deformation in amorphous solids. Phys Rev E, 1998, 57: 7192-7205

30 Wright W J, Hufnagel T C, Nix W D. Free volume coalescence and void formation in shear bands in metallic glass. J Appl Phys, 2003, 93: $1432-1437$

31 Zhao M, Li M. Interpreting the change in shear band inclination angle in metallic glasses. Appl Phys Lett, 2008, 93: 241906

32 Xiao Y H, Wu Y, Liu Z Y, et al. Effects of cooling rates on the mechanical properties of a Ti-based bulk metallic glass. Sci China Phys Mech Astron, 2010, 53: 394-398 